\title{
Robust Secrecy Beamforming for MIMO SWIPT with Probabilistic Constraints
}

\author{
Muhammad R. A. Khandaker and Kai-Kit Wong \\ Department of Electronic and Electrical Engineering \\ University College London \\ Gower Street, London, WC1E 7JE, United Kingdom \\ e-mail: \{m.khandaker, kai-kit.wong\}@ucl.ac.uk
}

\begin{abstract}
This paper considers simultaneous wireless information and power transfer (SWIPT) in a multiple-input multipleoutput (MIMO) wiretap channel with energy harvesting receivers. The main objective is to keep the probability of the legitimate user's achievable secrecy rate outage as well as the energy receivers' harvested energy outage as caused by CSI uncertainties below given thresholds. This probabilisticconstrained secrecy rate maximization problem presents a significant analytical and computational challenge since any closedform for the probabilistic constraints with $\log$ - det functions is intractable. In this paper, we address this challenging issue using convex restrictions. In particular, we derive decompositionbased large deviation inequalities to transform the probabilistic constraints into second-order cone (SOC) constraints which are easier to handle. Then we show that a robust safe solution can be obtained through solving two convex sub-problems in an alternating fashion.
\end{abstract}

\section{INTRODUCTION}

One of the game changing technologies in wireless communications that attracted booming research interests over the past decade is the concept of wireless energy harvesting. Enough have been spoken about its prospects, and numerous technologies have been proposed in the literature to harvest the maximum benefits of energy harvesting (see, e.g., [1] and the references therein). Since RF signals can transport information as well as energy simultaneously, mobile devices have access to both energy and data at the same time. All that they need to do is to harvest the energy brought to their door-step opportunistically.

However, a fundamental challenge in designing optimal transmit precoders for simultaneous wireless information and power transfer (SWIPT) systems is the diverse power sensitivity level at which the information and energy receiver circuits operate (e.g., $-10 \mathrm{dBm}$ for energy receivers (ERs) versus $-60 \mathrm{dBm}$ for information receivers (IRs)). Another practical challenge against RF energy harvesting is that the energy carried by RF signals decays drastically with increasing distance due to propagation path loss. Exploiting spatial diversity techniques, multiple-input multiple-output (MIMO) systems can help to circumvent these challenges to some extent [2]-[4].

Another potential strategy proposed in the literature to improve energy harvesting efficiency in SWIPT systems is the receiver-location based scheduling scheme [2], [5], in which the receivers located in closer proximity to the transmitter are scheduled for harvesting energy, whereas those far from the transmitter are scheduled for decoding information. Although the scheme apparently seems to be beneficial for energy harvesting (EH), actually it gives rise to an undesired security vulnerability for transmitting secret information in scenarios where ERs are supposed to be kept in the dark about the secret message. Specifically, ERs in the scheme have better fading channels than IRs and thus have higher probability to successfully decode the information sent to the IRs [6], [7]. Therefore, SWIPT systems deserve efficient measures in order to be able to successfully transfer secret messages keeping the ERs ignorant of the secret message to the IR.

In order to improve information security is wireless systems, physical-layer security approaches are being widely considered in the information-theoretic society since the prominent technique can afford an extra security layer on top of the traditional cryptographic approaches. Such measures can make SWIPT more significant in practice. Hence physical-layer security techniques have recently been introduced for SWIPT in numerous scenarios [6]-[12]. While it is generally expected that full channel state information (CSI) of all the links be available at the transmitter in order to fully exploit the spatial degrees of freedom (DoF) provided by MIMO technology, it is generally very unrealistic in practice. In particular, it is almost impossible to obtain perfect eavesdroppers' CSI since eavesdroppers are often unknown malicious agents. The situation can further worsen if multiple eavesdroppers collude together in an attempt to maximize their interception through joint receive beamforming. Hence the authors in [7], [9] considered robust design based on deterministic channel uncertainty models for SWIPT in scenarios where the ERs may collude together, as opposed to perfect CSI assumption for non-colluding eavesdroppers in [6].

Other recent works with secrecy in SWIPT either considered worst-case robust approach in which the CSI errors are assumed to be within a bounded set, or correlation-based approach in which the channel statistics is available. A delaylimited secrecy SWIPT system has been considered in [8] in which all the nodes are equipped with a single antenna. A randomization-guided rank-one suboptimal solution has been proposed in [10] for worst-case MISO secrecy SWIPT systems, and in [11] for MIMO SWIPT systems. Worst-case based MISO secrecy SWIPT optimization has also been considered in [12] for norm-bounded channel uncertainty model 
based on successive convex approximation technique.

However, due to inaccurate channel estimation methods, often it may not be possible to obtain these deterministic models perfectly. In such cases, secrecy as well as energy harvesting outage is an obvious effect. Hence in this paper, we focus on probabilistically robust design of secrecy rate optimization for MIMO systems in presence of a multi-antenna eavesdropper (MIMOME) as well as energy harvesters. Note that in contrast to the deterministic models in existing literature, this formulation provides a safe performance guarantee up to certain QoS requirements.

As is well known, the secrecy rate outage constraints in MIMOME systems present a significant analytical and computational challenge since no closed-form expression exists for the probabilistic constraints with matrix variables. The inclusion of the energy harvesting outage constraints only thrives that challenge. As such, a common practice in the robust optimization literature is to develop safe tractable approximations of the outage constraints that are computationally efficient and are good in accuracies. In this paper, we first transformed the probabilistic constraints involving log and det functions, which are generated by the MIMOME configuration, into tractable second-order cone (SOC) constraints. Then we showed that a robust safe solution can be obtained through solving two convex sub-problems in an alternating fashion.

Notations: Throughout the paper we use the following notation standards. Boldface lowercase and uppercase letters are used to represent vectors and matrices, respectively. The symbol $\mathbf{I}_{n}$ denotes an $n \times n$ identity matrix, $\mathbf{0}$ is a zero vector or matrix. Also, $\mathbf{A}^{H}, \operatorname{tr}(\mathbf{A}), \operatorname{rank}(\mathbf{A})$, and $|\mathbf{A}|$ represent the Hermitian (conjugate) transpose, trace, rank and determinant of a matrix $\mathbf{A} ; \operatorname{Pr}[\cdot]$ represents the probability of an event; $\|\cdot\|$ and $\|\cdot\|_{F}$ represent the Euclidean norm and Frobenius norm, respectively; $\mathbf{A} \succeq \mathbf{0}(\mathbf{A} \succ \mathbf{0})$ means that $\mathbf{A}$ is a Hermitian positive semidefinite (definite) matrix. The notation $\mathbf{x} \sim \mathcal{C N}(\boldsymbol{\mu}, \boldsymbol{\Sigma})$ means that $\mathbf{x}$ is a random vector following a complex circularly symmetric Gaussian distribution with the mean vector $\boldsymbol{\mu}$ and the covariance matrix of $\boldsymbol{\Sigma}$.

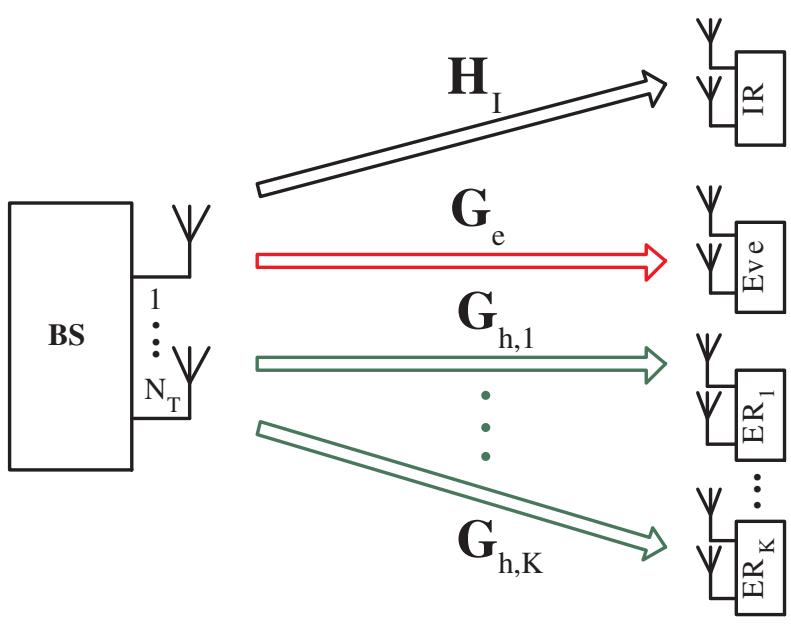

Fig. 1. A MIMOME wiretap system with energy harvesting nodes.

\section{System ModeL}

A MIMO downlink system is considered for SWIPT in presence of a multi-antenna eavesdropper, as illustrated in Fig. 1. All the nodes are equipped with multiple antennas. The base station (BS) aims to transmit information to a legitimate user (IR) and energy to $K$ energy harvesting (EH) receivers keeping the information secret from the eavesdropper (Eve). The BS, IR, Eve, and the $k$ th ER are equipped with $N_{\mathrm{T}}>1$, $N_{\mathrm{d}}, N_{\mathrm{e}}$, and $N_{\mathrm{h}, k}$ antennas, respectively. The BS performs linear transmit precoding to send secret information to the IR. By letting $\mathrm{x}$ be the transmit signal vector, the received signals at the IR, the Eve and the $k$ th ER can be modeled, respectively, as

$$
\begin{aligned}
\mathbf{y}_{\mathrm{d}} & =\mathbf{H}_{\mathrm{d}}^{H} \mathbf{x}+\mathbf{n}_{\mathrm{d}}, \\
\mathbf{y}_{\mathrm{e}} & =\mathbf{G}_{\mathrm{e}}^{H} \mathbf{x}+\mathbf{n}_{\mathrm{e}}, \\
\mathbf{y}_{\mathrm{h}, k} & =\mathbf{G}_{\mathrm{h}, k}^{H} \mathbf{x}+\mathbf{n}_{\mathrm{h}, k}, \text { for } k=1, \ldots, K,
\end{aligned}
$$

where $\mathbf{H}_{\mathrm{d}}, \mathbf{G}_{\mathrm{e}}$, and $\mathbf{G}_{\mathrm{h}, k}$ are the conjugated complex channel matrices between the BS and the IR, the Eve, and the $k$ th ER, respectively, $\mathbf{n}_{\mathrm{d}} \sim \mathcal{C N}\left(\mathbf{0}, \sigma_{\mathrm{d}}^{2} \mathbf{I}_{N_{\mathrm{d}}}\right), \mathbf{n}_{\mathrm{e}} \sim \mathcal{C N}\left(\mathbf{0}, \sigma_{\mathrm{e}}^{2} \mathbf{I}_{N_{\mathrm{e}}}\right)$, and $\mathbf{n}_{\mathrm{h}, k} \sim \mathcal{C N}\left(\mathbf{0}, \sigma_{\mathrm{h}}^{2} \mathbf{I}_{N_{\mathrm{h}, k}}\right)$ are the additive Gaussian noises at the IR, the Eve, and the $k$ th ER, respectively. The BS chooses the transmit signal vector $\mathbf{x} \sim \mathcal{C N}\left(\mathbf{0}, \mathbf{Q}_{\mathrm{I}}\right)$ where $\mathbf{Q}_{\mathrm{I}} \succeq \mathbf{0}$ is the transmit covariance matrix. Thus the mutual information (MI) between the BS and the IR is given by [13]

$$
C_{\mathrm{I}}\left(\mathbf{Q}_{\mathrm{I}}\right)=\log \left|\mathbf{I}_{N_{\mathrm{d}}}+\frac{1}{\sigma_{\mathrm{d}}^{2}} \mathbf{H}_{\mathrm{d}}^{H} \mathbf{Q}_{\mathrm{I}} \mathbf{H}_{\mathrm{d}}\right|,
$$

and that between the BS and the eavesdropper is given by

$$
C_{\mathrm{e}}\left(\mathbf{Q}_{\mathrm{I}}\right)=\log \left|\mathbf{I}_{N_{\mathrm{e}}}+\frac{1}{\sigma_{\mathrm{e}}^{2}} \mathbf{G}_{\mathrm{e}}^{H} \mathbf{Q}_{\mathrm{I}} \mathbf{G}_{\mathrm{e}}\right| .
$$

Accordingly, the achievable secrecy rate at the IR is given by [13]

$$
R_{\mathrm{S}}=\left[C_{\mathrm{I}}\left(\mathbf{Q}_{\mathrm{I}}\right)-C_{\mathrm{e}}\left(\mathbf{Q}_{\mathrm{I}}\right)\right]^{+},
$$

where $\{a\}^{+}$indicates the $\max (0, a)$. Note that (6) gives the perfect secrecy rate when the IR can correctly decode the confidential information at $R_{\mathrm{S}}$ bits per channel use, while Eve can retrieve almost nothing.

The harvested power at the $k$ th ER is given by

$$
E_{k}=\xi_{k} \operatorname{tr}\left(\mathbf{G}_{\mathrm{h}, k}^{H} \mathbf{Q}_{\mathbf{I}} \mathbf{G}_{\mathrm{h}, k}\right),
$$

where $\xi_{k} \in(0,1]$ is the energy conversion efficiency of the energy transducers at the $k$ th ER. For simplicity, it is also assumed that the harvested energy due to the environmental noise at the ERs is negligible [2].

\section{Robust Optimization with Probabilistic CONSTRAINTS}

In most of the existing works with secrecy SWIPT, it is assumed that the instantaneous CSI of all the receivers (including Eve) is available at the transmitter. However, in practical wireless communication systems, perfect CSI of the eavesdropper is always challenging to obtain and an important 
issue is how to robustify a secure transmit design in the presence of imperfect CSI. Hence in this section, our endeavour is to develop a probabilistically robust algorithm for the secrecy rate maximization problem with QoS outage constraints. We assume that the BS has incomplete knowledge of ERs' as well as Eve's channels while the IR's channel is perfectly known.

We consider the widely used Gaussian channel error model for the imperfect CSI. We assume that the channel error matrices have circularly symmetric complex Gaussian (CSCG) distribution. Thus, the actual channels between the BS and the ERs can be modeled as

$$
\mathbf{G}_{\mathrm{h}, k}=\hat{\mathbf{G}}_{\mathrm{h}, k}+\boldsymbol{\Delta}_{\mathrm{h}, k}, \text { for } k=1, \cdots, K,
$$

where $\hat{\mathbf{G}}_{\mathrm{h}, k} \in \mathbb{C}^{N_{\mathrm{T}} \times N_{\mathrm{h}, k}}$ is the estimated CSI of the $k$-th ER and $\boldsymbol{\Delta}_{\mathrm{h}, k} \in \mathbb{C}^{N_{\mathrm{T}} \times N_{\mathrm{h}, k}}$, for $k=1, \ldots, K$, represent the channel uncertainties such that $\boldsymbol{\delta}_{\mathrm{h}, k} \triangleq \operatorname{vec}\left(\boldsymbol{\Delta}_{\mathrm{h}, k}\right) \sim$ $\mathcal{C N}\left(\mathbf{0}, \mathbf{R}_{\mathrm{h}, k}\right), \mathbf{R}_{\mathrm{h}, k}$ is a PSD matrix. Similarly, the Eves' channel uncertainty model can be represented by

$$
\mathbf{G}_{\mathrm{e}}=\hat{\mathbf{G}}_{\mathrm{e}}+\boldsymbol{\Delta}_{\mathrm{e}}
$$

where $\hat{\mathbf{G}}_{\mathrm{e}} \in \mathbb{C}^{N_{\mathrm{T}} \times N_{\mathrm{e}}}$ is the estimated CSI of Eve and $\boldsymbol{\Delta}_{\mathrm{e}} \in \mathbb{C}^{N_{\mathrm{T}} \times N_{\mathrm{e}}}$ represent the channel uncertainties such that $\boldsymbol{\delta}_{\mathrm{e}} \triangleq \operatorname{vec}\left(\boldsymbol{\Delta}_{\mathrm{e}}\right) \sim \mathcal{C N}\left(\mathbf{0}, \mathbf{R}_{\mathrm{e}}\right), \mathbf{R}_{\mathrm{e}}$ is a PSD matrix. Thus, the secrecy rate maximization problem with probabilistic QoS constraints can be formulated as

$$
\begin{array}{rl}
\max _{R, \mathbf{Q}_{\mathrm{I}}} & R \\
\text { s.t. } & \operatorname{Pr}\left\{C_{\mathrm{I}}\left(\mathbf{Q}_{\mathrm{I}}\right)-C_{\mathrm{e}}\left(\mathbf{Q}_{\mathrm{I}}\right) \geq R\right\} \geq 1-p, \\
& \operatorname{Pr}\left[E_{k} \geq \eta_{k}\right] \geq 1-q, \forall k, \\
& \operatorname{tr}\left(\mathbf{Q}_{\mathrm{I}}\right) \leq P_{\mathrm{T}} \\
& \mathbf{Q}_{\mathrm{I}} \succeq \mathbf{0}, \quad R \geq 0 .
\end{array}
$$

To the best of our knowledge, the above problem formulation with secrecy rate and energy harvesting outage constraints for the MIMOME wiretap channel has not been considered in any existing work. Note that the problem formulation in (10) guarantees that the IR can successfully decode its message at least $(1-p) \times 100 \%$ of the time. Similarly, the ERs can harvest the minimum required amount of power at least $(1-q) \times 100 \%$ of the time.

Our next endeavour is to find tractable convex restrictions for the probabilistic constraints. Two key challenges we need to tackle in order to derive any such restriction are the probability operator and the $\log -\operatorname{det}$ function in the Eve's MI expression. Based on Fenchel conjugate arguments, the following lemma can be defined from [14]:

Lemma 1. Given any integer $n$ and positive-definite matrix $(\mathbf{D} \succ \mathbf{0}) \in \mathbb{C}^{n \times n}$. Considering the function $f(\mathbf{S}, \mathbf{D})=$ $-\operatorname{tr}(\mathbf{S D})+\log |\mathbf{S}|+n$, it holds true that

$$
\max _{\mathbf{S} \in \mathbb{C}^{n \times n}, \mathbf{S} \succeq \mathbf{0}} f(\mathbf{S}, \mathbf{D})=\log \left|\mathbf{D}^{-1}\right| .
$$

The merit of Lemma 1 is that it introduces an auxiliary variable and expresses the original complicated $\log$ - det functions in terms of a matrix trace function and the new variable. Hence to make the probabilistic secrecy constraint (10b) more tractable, we apply Lemma 1 and rewrite $C_{\mathrm{e}}\left(\mathbf{Q}_{\mathrm{I}}\right)$ as

$$
\begin{aligned}
C_{\mathrm{e}}\left(\mathbf{Q}_{\mathrm{I}}\right) & =-\log \left|\left(\mathbf{I}_{N_{\mathrm{e}}}+\frac{1}{\sigma_{\mathrm{e}}^{2}} \mathbf{G}_{\mathrm{e}}^{H} \mathbf{Q}_{\mathrm{I}} \mathbf{G}_{\mathrm{e}}\right)^{-1}\right| \\
& =-\max _{\tilde{\mathbf{Q}}_{\mathbf{I}} \succeq \mathbf{0}} f\left(\tilde{\mathbf{Q}}_{\mathrm{I}}, \mathbf{I}_{N_{\mathrm{e}}}+\frac{1}{\sigma_{\mathrm{e}}^{2}} \mathbf{G}_{\mathrm{e}}^{H} \mathbf{Q}_{\mathrm{I}} \mathbf{G}_{\mathrm{e}}\right) \\
& =\min _{\tilde{\mathbf{Q}}_{\mathrm{I}} \succeq \mathbf{0}}-f\left(\tilde{\mathbf{Q}}_{\mathrm{I}}, \mathbf{I}_{N_{\mathrm{e}}}+\frac{1}{\sigma_{\mathrm{e}}^{2}} \mathbf{G}_{\mathrm{e}}^{H} \mathbf{Q}_{\mathrm{I}} \mathbf{G}_{\mathrm{e}}\right) .
\end{aligned}
$$

In order to simplify the notations, let us now denote

$$
\begin{aligned}
& \bar{f}\left(\tilde{\mathbf{Q}}_{\mathrm{I}}, \mathbf{Q}_{\mathrm{I}}\right) \triangleq-f\left(\tilde{\mathbf{Q}}_{\mathrm{I}}, \mathbf{I}_{N_{\mathrm{e}}}+\frac{1}{\sigma_{\mathrm{e}}^{2}} \mathbf{G}_{\mathrm{e}}^{H} \mathbf{Q}_{\mathrm{I}} \mathbf{G}_{\mathrm{e}}\right) \\
& =\operatorname{tr}\left(\left(\mathbf{I}_{N_{\mathrm{e}}}+\frac{1}{\sigma_{\mathrm{e}}^{2}} \mathbf{G}_{\mathrm{e}}^{H} \mathbf{Q}_{\mathrm{I}} \mathbf{G}_{\mathrm{e}}\right) \tilde{\mathbf{Q}}_{\mathrm{I}}\right)-\log \left|\tilde{\mathbf{Q}}_{\mathrm{I}}\right|-N_{\mathrm{e}},
\end{aligned}
$$

where the last equality is obtained from Lemma 1 . Now by substituting (12) back into the secrecy rate outage constraint (10b), we obtain

$$
\begin{aligned}
& \operatorname{Pr}\left\{C_{\mathrm{d}}\left(\mathbf{Q}_{\mathrm{I}}\right)-C_{\mathrm{e}}\left(\mathbf{Q}_{\mathrm{I}}\right) \geq R\right\} \geq 1-p \\
& \Longleftrightarrow \operatorname{Pr}\left\{\min _{\tilde{\mathbf{Q}}_{\mathrm{I}} \succeq \mathbf{0}} \bar{f}\left(\tilde{\mathbf{Q}}_{\mathrm{I}}, \mathbf{Q}_{\mathrm{I}}\right) \leq C_{\mathrm{I}}\left(\mathbf{Q}_{\mathrm{I}}\right)-R\right\} \geq 1-p \\
& \Longleftrightarrow \operatorname{Pr}\left\{\bar{f}\left(\tilde{\mathbf{Q}}_{\mathrm{I}}, \mathbf{Q}_{\mathrm{I}}\right) \leq C_{\mathrm{I}}\left(\mathbf{Q}_{\mathrm{I}}\right)-R\right\} \geq 1-p, \\
& \quad \text { for some } \tilde{\mathbf{Q}}_{\mathrm{I}} \succeq \mathbf{0}, \\
& \Longleftrightarrow \operatorname{Pr}\left\{\operatorname{tr}\left(\mathbf{G}_{\mathrm{e}}^{H} \mathbf{Q}_{\mathrm{I}} \mathbf{G}_{\mathrm{e}} \tilde{\mathbf{Q}}_{\mathrm{I}}\right) \leq \tau\right\} \geq 1-p,
\end{aligned}
$$

where $\tau \triangleq \sigma_{\mathrm{e}}^{2}\left(C_{\mathrm{I}}\left(\mathbf{Q}_{\mathrm{I}}\right)-R+\log \left|\tilde{\mathbf{Q}}_{\mathrm{I}}\right|+N_{\mathrm{e}}-\operatorname{tr}\left(\tilde{\mathbf{Q}}_{\mathrm{I}}\right)\right)$. Note that the constraint is still intractable due to the probability function. In the following, we focus on developing a safe approximation approach such that the probabilistic constraint could be satisfied through solving some convex constraints.

Applying the matrix identity $\operatorname{tr}\left(\mathbf{A}^{H} \mathbf{B C D}\right)=$ $\operatorname{vec}(\mathbf{A})^{H}\left(\mathbf{D}^{T} \otimes \mathbf{B}\right) \operatorname{vec}(\mathbf{C})$ we obtain

$$
\operatorname{tr}\left(\mathbf{G}_{\mathrm{e}}^{H} \mathbf{Q}_{\mathrm{I}} \mathbf{G}_{\mathrm{e}} \tilde{\mathbf{Q}}_{\mathrm{I}}\right)=\mathbf{g}_{\mathrm{e}}^{H}\left(\tilde{\mathbf{Q}}_{\mathrm{I}}^{T} \otimes \mathbf{Q}_{\mathrm{I}}\right) \mathbf{g}_{\mathrm{e}} .
$$

Then substituting $\mathbf{G}_{\mathrm{e}}=\hat{\mathbf{G}}_{\mathrm{e}}+\boldsymbol{\Delta}_{\mathrm{e}}$, i.e., $\mathrm{g}_{\mathrm{e}}=\hat{\mathrm{g}}_{\mathrm{e}}+\boldsymbol{\delta}_{\mathrm{e}}$, in equation (13) yields

$$
\begin{array}{r}
\operatorname{Pr}\left\{\boldsymbol { \delta } _ { \mathrm { e } } ^ { H } \left(\tilde{\mathbf{Q}}_{\mathrm{I}}^{T} \otimes\right.\right. \\
\left.\mathbf{Q}_{\mathrm{I}}\right) \boldsymbol{\delta}_{\mathrm{e}}+2 \Re\left\{\boldsymbol{\delta}_{\mathrm{e}}^{H}\left(\tilde{\mathbf{Q}}_{\mathrm{I}}^{T} \otimes \mathbf{Q}_{\mathrm{I}} \hat{\mathbf{g}}_{\mathrm{e}}\right)\right\} \\
\left.+\hat{\mathbf{g}}_{\mathrm{e}}^{H}\left(\tilde{\mathbf{Q}}_{\mathrm{I}}^{T} \otimes \mathbf{Q}_{\mathrm{I}}\right) \hat{\mathbf{g}}_{\mathrm{e}} \leq \tau\right\} \geq 1-p, \forall i
\end{array}
$$

where $\hat{\mathrm{g}}_{\mathrm{e}} \triangleq \operatorname{vec}\left(\hat{\mathbf{G}}_{\mathrm{e}}\right)$. Since $\boldsymbol{\delta}_{\mathrm{e}} \sim \mathcal{C N}\left(\mathbf{0}, \mathbf{R}_{\mathrm{e}}\right), \boldsymbol{\delta}_{\mathrm{e}}$ can be re-expressed as $\boldsymbol{\delta}_{\mathrm{e}}=\mathbf{R}_{\mathrm{e}}^{\frac{1}{2}} \mathbf{v}_{\mathrm{e}}$ such that $\mathbf{v}_{\mathrm{e}} \sim \mathcal{C N}\left(\mathbf{0}, \mathbf{I}_{N_{\mathrm{T}} N_{\mathrm{e}}}\right)$. Thus (15) can be re-expressed as

$$
\begin{aligned}
& \operatorname{Pr}\left[\mathbf{v}_{\mathrm{e}}^{H}\left[-\mathbf{R}_{\mathrm{e}}^{\frac{1}{2}}\left(\tilde{\mathbf{Q}}_{\mathrm{I}}^{T} \otimes \mathbf{Q}_{\mathrm{I}}\right) \mathbf{R}_{\mathrm{e}}^{\frac{1}{2}}\right] \mathbf{v}_{\mathrm{e}}+2 \Re\left\{\mathbf { v } _ { \mathrm { e } } ^ { H } \left[-\mathbf{R}_{\mathrm{e}}^{\frac{1}{2}}\right.\right.\right. \\
& \left.\left.\left.\left(\tilde{\mathbf{Q}}_{\mathrm{I}}^{T} \otimes \mathbf{Q}_{\mathrm{I}}\right) \hat{\mathbf{g}}_{\mathrm{e}}\right]\right\}-\hat{\mathbf{g}}_{\mathrm{e}}^{H}\left(\tilde{\mathbf{Q}}_{\mathrm{I}}^{T} \otimes \mathbf{Q}_{\mathrm{I}}\right) \hat{\mathbf{g}}_{\mathrm{e}}+\tau \geq 0\right] \geq 1-p .
\end{aligned}
$$


On the other hand, substituting the ERs' channel uncertainty models in the energy harvesting outage constraint (10c), we obtain

$$
\begin{aligned}
\operatorname{Pr}\left[\xi_{k} \operatorname{tr}\right. & \left.\left(\mathbf{G}_{\mathrm{h}, k}^{H} \mathbf{Q}_{\mathbf{I}} \mathbf{G}_{\mathrm{h}, k}\right) \geq \eta_{k}\right] \geq 1-q, \\
\Longleftrightarrow & \operatorname{Pr}\left[\operatorname { t r } \left(\boldsymbol{\Delta}_{\mathrm{h}, k}^{H} \mathbf{Q}_{\mathbf{I}} \boldsymbol{\Delta}_{\mathrm{h}, k}+\boldsymbol{\Delta}_{\mathrm{h}, k}^{H} \mathbf{Q}_{\mathbf{I}} \hat{\mathbf{G}}_{\mathrm{h}, k}+\hat{\mathbf{G}}_{\mathrm{h}, k}^{H} \mathbf{Q}_{\mathbf{I}}\right.\right. \\
& \left.\left.\times \boldsymbol{\Delta}_{\mathrm{h}, k}+\hat{\mathbf{G}}_{\mathrm{h}, k}^{H} \mathbf{Q}_{\mathbf{I}} \hat{\mathbf{G}}_{\mathrm{h}, k}\right) \geq \frac{\eta_{k}}{\xi_{k}}\right] \geq 1-q, \forall k . \quad(17)
\end{aligned}
$$

Applying the matrix identity $\operatorname{tr}\left(\mathbf{A}^{H} \mathbf{B C D}\right)=\operatorname{vec}(\mathbf{A})^{H}\left(\mathbf{D}^{T} \otimes\right.$ $\mathbf{B}) \operatorname{vec}(\mathbf{C})$, (17) can be re-expressed as

$$
\begin{gathered}
\operatorname{Pr}\left[\boldsymbol{\delta}_{\mathrm{h}, k}^{H}\left(\mathbf{I}_{N_{\mathrm{h}, k}} \otimes \mathbf{Q}_{\mathrm{I}}\right) \boldsymbol{\delta}_{\mathrm{h}, k}+2 \Re\left\{\boldsymbol{\delta}_{\mathrm{h}, k}^{H}\left(\mathbf{I}_{N_{\mathrm{h}, k}} \otimes \mathbf{Q}_{\mathrm{I}}\right) \hat{\mathbf{g}}_{\mathrm{h}, k}\right\}\right. \\
\left.+\hat{\mathbf{g}}_{\mathrm{h}, k}^{H}\left(\mathbf{I}_{N_{\mathrm{h}, k}} \otimes \mathbf{Q}_{\mathrm{I}}\right) \hat{\mathbf{g}}_{\mathrm{h}, k} \geq \frac{\eta_{k}}{\xi_{k}}\right] \geq 1-q, \forall k .
\end{gathered}
$$

Substituting $\boldsymbol{\delta}_{\mathrm{h}, k}=\mathbf{R}_{\mathrm{h}, k}^{\frac{1}{2}} \mathbf{u}_{\mathrm{h}, k}$ in (18) with $\mathbf{u}_{\mathrm{h}, k} \sim$ $\mathcal{C N}\left(\mathbf{0}, \mathbf{I}_{N_{\mathrm{T}} N_{\mathrm{h}, k}}\right)$, we obtain

$\operatorname{Pr}\left[\mathbf{u}_{\mathrm{h}, k}^{H} \mathbf{R}_{\mathrm{h}, k}^{\frac{1}{2}}\left(\mathbf{I}_{N_{\mathrm{h}, k}} \otimes \mathbf{Q}_{\mathrm{I}}\right) \mathbf{R}_{\mathrm{h}, k}^{\frac{1}{2}} \mathbf{u}_{\mathrm{h}, k}+2 \Re\left\{\mathbf{u}_{\mathrm{h}, k}^{H} \mathbf{R}_{\mathrm{h}, k}^{\frac{1}{2}}\left(\mathbf{I}_{N_{\mathrm{h}, k}}\right.\right.\right.$

$\left.\left.\left.\otimes \mathbf{Q}_{\mathrm{I}}\right) \hat{\mathbf{g}}_{\mathrm{h}, k}\right\}+\hat{\mathbf{g}}_{\mathrm{h}, k}^{H}\left(\mathbf{I}_{N_{\mathrm{h}, k}} \otimes \mathbf{Q}_{\mathrm{I}}\right) \hat{\mathbf{g}}_{\mathrm{h}, k}-\frac{\eta_{k}}{\xi_{k}} \geq 0\right] \geq 1-q, \forall k$.

At this point, one can notice that the probabilistic constraints (16) and (19) are of the form:

$$
\operatorname{Pr}\left\{\mathbf{x}^{H} \mathbf{A} \mathbf{x}+2 \Re\left\{\mathbf{x}^{H} \mathbf{r}\right\}+s \geq 0\right\} \geq 1-\rho,
$$

where $\mathbf{x} \sim \mathcal{C N}\left(\mathbf{0}, \mathbf{I}_{n}\right)$ is a standard complex Gaussian random vector, $\mathbf{A} \in \mathbb{H}^{n \times n}$ and $\mathbf{r} \in \mathbb{C}^{n}$ is a complex random vector. In order to develop some convex restrictions to tackle these probabilistic constraints, our aim is to find a convex function $g(\mathbf{A}, \mathbf{r}, s)$, such that

$$
\operatorname{Pr}\left\{\mathbf{x}^{H} \mathbf{A} \mathbf{x}+2 \Re\left\{\mathbf{x}^{H} \mathbf{r}\right\}+s \geq 0\right\} \leq g(\mathbf{A}, \mathbf{r}, s) .
$$

Then we will immediately have the following implication from [15]

$$
\begin{array}{r}
g(\mathbf{A}, \mathbf{r}, s) \leq \rho \Longrightarrow \operatorname{Pr}\left\{\mathbf{x}^{H} \mathbf{A} \mathbf{x}+2 \Re\left\{\mathbf{x}^{H} \mathbf{r}\right\}+s \geq 0\right\} \\
\geq 1-\rho .
\end{array}
$$

Thus the L.H.S. of the implication in (20) gives a safe approximation, which is convex, of the generally intractable probabilistic constraint in the R.H.S.

Note that such convex restriction approaches have also been followed in [16], [17] for MIMOME systems without considering the energy harvesting outage constraints based on Bernstein-type inequality (BTI) [18]. However, the BTI-based approach transforms the chance-constrained optimization problem into SDPs, the resulting safe designs are polynomial-time solvable [19]. Meanwhile, the SDPs can be very expensive to solve if the size of the probabilistic constraints in (10) is sufficiently large. Hence in this paper, we aim at developing convex restrictions involving simpler conic constraints. The method follows decomposition-based large deviation inequality (LDI) [20] for complex Gaussian quadratic functions as defined in the following lemma:

Lemma 2. [15, Lemma 2] Let $\mathbf{x} \sim \mathcal{C N}\left(\mathbf{0}, \mathbf{I}_{n}\right)$ be a standard complex Gaussian random vector, and let $\mathbf{A} \in \mathbb{H}^{n \times n}$ and $\mathbf{r} \in$ $\mathbb{C}^{n}$ be given. Then, for any $\nu>\frac{1}{\sqrt{2}}$ and $\zeta>0$, we have

$$
\begin{aligned}
\operatorname{Pr} & \left\{\mathbf{x}^{H} \mathbf{A x}+2 \operatorname{Re}\left\{\mathbf{x}^{H} \mathbf{r}\right\} \leq \operatorname{tr}(\mathbf{A})-\zeta\right\} \\
& \leq \begin{cases}\exp \left(-\frac{\zeta^{2}}{4 T^{2}}\right) & \text { for } 0<\zeta \leq 2 \bar{\nu} \nu T, \\
\exp \left(-\frac{\bar{\nu} \nu \zeta}{T}+(\bar{\nu} \nu)^{2}\right) & \text { for } \zeta>2 \bar{\nu} \nu T,\end{cases}
\end{aligned}
$$

where $\bar{\nu}=1-\frac{1}{2 \nu^{2}}, \quad T=\nu\|\mathbf{A}\|_{F}+\frac{1}{\sqrt{2}}\|\mathbf{r}\|$.

It is interesting to observe that Lemma 2 has actually decomposed the sum of dependent random variables $\left(\mathbf{x}^{H} \mathbf{A x}+\right.$ $\left.2 \operatorname{Re}\left\{\mathbf{x}^{H} \mathbf{r}\right\}\right)$ into sums of independent random variables. This idea has been widely used in the literature of probability theory; see, e.g., [20], [21].

Next, we concentrate on deriving convex restrictions of (16) and (17) based on the LDI approach. To derive a convex restriction of (16) using Lemma 2, we set

$$
\begin{aligned}
& \zeta_{\mathrm{e}}=\operatorname{tr}\left(-\mathbf{R}_{\mathrm{e}}^{\frac{1}{2}}\left(\tilde{\mathbf{Q}}_{\mathrm{I}}^{T} \otimes \mathbf{Q}_{\mathrm{I}}\right) \mathbf{R}_{\mathrm{e}}^{\frac{1}{2}}\right)+\tau_{\mathrm{e}}, \\
& T_{\mathrm{e}}=\nu\left\|-\mathbf{R}_{\mathrm{e}}^{\frac{1}{2}}\left(\tilde{\mathbf{Q}}_{\mathrm{I}}^{T} \otimes \mathbf{Q}_{\mathrm{I}}\right) \mathbf{R}_{\mathrm{e}}^{\frac{1}{2}}\right\|_{F} \\
& +\frac{1}{\sqrt{2}}\left\|-\mathbf{R}_{\mathrm{e}}^{\frac{1}{2}}\left(\tilde{\mathbf{Q}}_{\mathrm{I}}^{T} \otimes \mathbf{Q}_{\mathrm{I}}\right) \hat{\mathbf{g}}_{\mathrm{e}}\right\|,
\end{aligned}
$$

where $\tau_{\mathrm{e}} \triangleq-\hat{\mathbf{g}}_{\mathrm{e}}^{H}\left(\tilde{\mathbf{Q}}_{\mathrm{I}}^{T} \otimes \mathbf{Q}_{\mathrm{I}}\right) \hat{\mathbf{g}}_{\mathrm{e}}+\tau$, and choose $\nu>\frac{1}{\sqrt{2}}$ from the solution to the following quadratic equation

$$
\bar{\nu} \nu=\left(1-1 /\left(2 \nu^{2}\right)\right) \nu=\sqrt{-\ln (p)} .
$$

The resulting safe approximation can then be expressed as

$$
\operatorname{tr}\left(-\mathbf{R}_{\mathrm{e}}^{\frac{1}{2}}\left(\tilde{\mathbf{Q}}_{\mathrm{I}}^{T} \otimes \mathbf{Q}_{\mathrm{I}}\right) \mathbf{R}_{\mathrm{e}}^{\frac{1}{2}}\right)+\tau_{\mathrm{e}} \geq 2 \sqrt{-\ln (p)} T_{\mathrm{e}} .
$$

Replacing $T_{\mathrm{e}}$ by the appropriate expression, it is easy to verify that (25) can be expressed as a system of SOC constraints. In particular, we obtain the following convex restriction method for tackling the probabilistic constraint (16):

$$
\left\{\begin{aligned}
\operatorname{tr}\left(-\mathbf{R}_{\mathrm{e}}^{\frac{1}{2}}\left(\tilde{\mathbf{Q}}_{\mathrm{I}}^{T} \otimes \mathbf{Q}_{\mathrm{I}}\right) \mathbf{R}_{\mathrm{e}}^{\frac{1}{2}}\right)+\tau_{\mathrm{e}} & \geq 2 \sqrt{-\ln (p)} \\
& \times\left(\alpha_{\mathrm{e}}+\beta_{\mathrm{e}}\right), \\
\frac{1}{\sqrt{2}}\left\|-\mathbf{R}_{\mathrm{e}}^{\frac{1}{2}}\left(\tilde{\mathbf{Q}}_{\mathrm{I}}^{T} \otimes \mathbf{Q}_{\mathrm{I}}\right) \hat{\mathbf{g}}_{\mathrm{e}}\right\| & \leq \alpha_{\mathrm{e}}, \\
\nu\left\|-\mathbf{R}_{\mathrm{e}}^{\frac{1}{2}}\left(\tilde{\mathbf{Q}}_{\mathrm{I}}^{T} \otimes \mathbf{Q}_{\mathrm{I}}\right) \mathbf{R}_{\mathrm{e}}^{\frac{1}{2}}\right\|_{F} & \leq \beta_{\mathrm{e}},
\end{aligned}\right.
$$

where $\alpha_{\mathrm{e}}, \beta_{\mathrm{e}} \in \mathbb{R}$ are slack variables. Similarly, defining the slack variables $\psi_{\mathrm{h}, k}$ and $\omega_{\mathrm{h}, k}$, the energy harvesting outage constraint (17) can be expressed as

$$
\left\{\begin{array}{c}
\operatorname{tr}\left(\mathbf{R}_{\mathrm{h}, k}^{\frac{1}{2}}\left(\mathbf{I}_{N_{\mathrm{h}, k}} \otimes \mathbf{Q}_{\mathrm{I}}\right) \mathbf{R}_{\mathrm{h}, k}^{\frac{1}{2}}\right)+\hat{\mathbf{g}}_{\mathrm{h}, k}^{H}\left(\mathbf{I}_{N_{\mathrm{h}, k}} \otimes \mathbf{Q}_{\mathrm{I}}\right) \\
\quad \times \hat{\mathbf{g}}_{\mathrm{h}, k}-\frac{\eta_{k}}{\xi_{k}} \geq 2 \sqrt{-\ln (q)}\left(\psi_{\mathrm{h}, k}+\omega_{\mathrm{h}, k}\right), \\
\frac{1}{\sqrt{2}}\left\|\mathbf{R}_{\mathrm{h}, k}^{\frac{1}{2}}\left(\mathbf{I}_{N_{\mathrm{h}, k}} \otimes \mathbf{Q}_{\mathrm{I}}\right) \hat{\mathbf{g}}_{\mathrm{h}, k}\right\| \leq \psi_{\mathrm{h}, k}, \\
\nu\left\|\mathbf{R}_{\mathrm{h}, k}^{\frac{1}{2}}\left(\mathbf{I}_{N_{\mathrm{h}, k}} \otimes \mathbf{Q}_{\mathrm{I}}\right) \mathbf{R}_{\mathrm{h}, k}^{\frac{1}{2}}\right\|_{F} \leq \omega_{\mathrm{h}, k} .
\end{array}\right.
$$


Thus, applying the large deviation inequality method to the outage constrained problem (10), we obtain the convex restriction formulation of the secrecy rate maximization problem as

$$
\begin{aligned}
& \max _{\mathbf{Q}_{\mathrm{I}}, \tilde{\mathbf{Q}}_{\mathrm{I}}, R, \alpha_{\mathrm{e}}, \beta_{\mathrm{e}},\left\{\psi_{\mathrm{h}, k}\right\},\left\{\omega_{\mathrm{h}, k}\right\}} R \\
& \text { s.t. } \quad \operatorname{tr}\left(-\mathbf{R}_{\mathrm{e}}^{\frac{1}{2}}\left(\tilde{\mathbf{Q}}_{\mathrm{I}}^{T} \otimes \mathbf{Q}_{\mathrm{I}}\right) \mathbf{R}_{\mathrm{e}}^{\frac{1}{2}}\right)-\hat{\mathbf{g}}_{\mathrm{e}}^{H}\left(\tilde{\mathbf{Q}}_{\mathrm{I}}^{T} \otimes \mathbf{Q}_{\mathrm{I}}\right) \hat{\mathbf{g}}_{\mathrm{e}} \\
& \quad+\sigma_{\mathrm{e}}^{2}\left(C_{\mathrm{I}}\left(\mathbf{Q}_{\mathrm{I}}\right)-R+\log \left|\tilde{\mathbf{Q}}_{\mathrm{I}}\right|+N_{\mathrm{e}}-\operatorname{tr}\left(\tilde{\mathbf{Q}}_{\mathrm{I}}\right)\right) \\
& \quad \geq 2 \sqrt{-\ln (p)}\left(\alpha_{\mathrm{e}}+\beta_{\mathrm{e}}\right), \\
& \frac{1}{\sqrt{2}}\left\|-\mathbf{R}_{\mathrm{e}}^{\frac{1}{2}}\left(\tilde{\mathbf{Q}}_{\mathrm{I}}^{T} \otimes \mathbf{Q}_{\mathrm{I}}\right) \hat{\mathbf{g}}_{\mathrm{e}}\right\| \leq \alpha_{\mathrm{e}}, \\
& \nu\left\|\operatorname{vec}\left(-\mathbf{R}_{\mathrm{e}}^{\frac{1}{2}}\left(\tilde{\mathbf{Q}}_{\mathrm{I}}^{T} \otimes \mathbf{Q}_{\mathrm{I}}\right) \mathbf{R}_{\mathrm{e}}^{\frac{1}{2}}\right)\right\| \leq \beta_{\mathrm{e}}, \\
& \operatorname{tr}\left(\mathbf{R}_{\mathrm{h}, k}^{\frac{1}{2}}\left(\mathbf{I}_{N_{\mathrm{h}, k}} \otimes \mathbf{Q}_{\mathrm{I}}\right) \mathbf{R}_{\mathrm{h}, k}^{\frac{1}{2}}\right)+\hat{\mathbf{g}}_{k}^{H}\left(\mathbf{I}_{N_{\mathrm{h}, k}} \otimes \mathbf{Q}_{\mathrm{I}}\right) \hat{\mathbf{g}}_{k}-\frac{\eta_{k}}{\xi_{k}} \\
& \quad \geq 2 \sqrt{-\ln (q)}\left(\psi_{\mathrm{h}, k}+\omega_{\mathrm{h}, k}\right), \\
& \frac{1}{\sqrt{2}}\left\|\mathbf{R}_{\mathrm{h}, k}^{\frac{1}{2}}\left(\mathbf{I}_{N_{\mathrm{h}, k}} \otimes \mathbf{Q}_{\mathrm{I}}\right) \hat{\mathbf{g}}_{\mathrm{h}, k}\right\| \leq \psi_{\mathrm{h}, k}, \\
& \nu\left\|\operatorname{vec}\left(\mathbf{R}_{\mathrm{h}, k}^{\frac{1}{2}}\left(\mathbf{I}_{N_{\mathrm{h}, k}} \otimes \mathbf{Q}_{\mathrm{I}}\right) \mathbf{R}_{\mathrm{h}, k}^{\frac{1}{2}}\right)\right\| \leq \omega_{\mathrm{h}, k} \\
& \mathbf{Q}_{\mathrm{I}} \succeq \mathbf{0}, \quad\left\{R, \quad \alpha_{\mathrm{e}}, \beta_{\mathrm{e}}\right\} \geq 0, \quad\left\{\psi_{\mathrm{h}, k}, \omega_{\mathrm{h}, k}\right\} \geq 0 .
\end{aligned}
$$

Note that the above problem contains only SOC constraints in terms of $\mathbf{Q}_{\mathrm{I}}$ or $\tilde{\mathbf{Q}}_{\mathrm{I}}$ individually, but not jointly. Hence we solve the problem in an alternating fashion in order to find the optimal $\mathbf{Q}_{\mathrm{I}}$. In particular, it can be easily verified that for given $\mathbf{Q}_{\mathrm{I}}$ or $\mathbf{Q}_{\mathrm{I}}$, problem (27) is an SOCP with respect to the remaining variables. Thus for given $\tilde{\mathbf{Q}}_{\mathrm{I}}$, problem (27) can be solved using interior point methods [19]. Next, $\tilde{\mathbf{Q}}_{\mathrm{I}}$ is updated through solving the following problem with known $\mathbf{Q}_{\mathrm{I}}$ :

$$
\begin{aligned}
& \max _{\tilde{\mathbf{Q}}_{\mathrm{I}}, R, \alpha_{\mathrm{e}}, \beta_{\mathrm{e}}} R \\
& \text { s.t. } \quad \operatorname{tr}\left(-\mathbf{R}_{\mathrm{e}}^{\frac{1}{2}}\left(\tilde{\mathbf{Q}}_{\mathrm{I}}^{T} \otimes \mathbf{Q}_{\mathrm{I}}\right) \mathbf{R}_{\mathrm{e}}^{\frac{1}{2}}\right)-\hat{\mathbf{g}}_{\mathrm{e}}^{H}\left(\tilde{\mathbf{Q}}_{\mathrm{I}}^{T} \otimes \mathbf{Q}_{\mathrm{I}}\right) \hat{\mathbf{g}}_{\mathrm{e}} \\
& +\sigma_{\mathrm{e}}^{2}\left(C_{\mathrm{I}}\left(\mathbf{Q}_{\mathrm{I}}\right)-R+\log \left|\tilde{\mathbf{Q}}_{\mathrm{I}}\right|+N_{\mathrm{e}}-\operatorname{tr}\left(\tilde{\mathbf{Q}}_{\mathrm{I}}\right)\right) \\
& \geq 2 \sqrt{-\ln (p)}\left(\alpha_{\mathrm{e}}+\beta_{\mathrm{e}}\right), \\
& \frac{1}{\sqrt{2}}\left\|-\mathbf{R}_{\mathrm{e}}^{\frac{1}{2}}\left(\tilde{\mathbf{Q}}_{\mathrm{I}}^{T} \otimes \mathbf{Q}_{\mathrm{I}}\right) \hat{\mathbf{g}}_{\mathrm{e}}\right\| \leq \alpha_{\mathrm{e}}, \\
& \nu\left\|\operatorname{vec}\left(-\mathbf{R}_{\mathrm{e}}^{\frac{1}{2}}\left(\tilde{\mathbf{Q}}_{\mathrm{I}}^{T} \otimes \mathbf{Q}_{\mathrm{I}}\right) \mathbf{R}_{\mathrm{e}}^{\frac{1}{2}}\right)\right\| \leq \beta_{\mathrm{e}}, \\
& \left\{R, \alpha_{\mathrm{e}}, \beta_{\mathrm{e}}\right\} \geq 0 \text {. }
\end{aligned}
$$

Note that in problem (28), we have dropped the convex restriction constraints relevant to the energy harvesting outage requirements since those constraints are irrelevant to $\tilde{\mathbf{Q}}_{\mathrm{I}}$.

\section{Simulation Results}

In this section, we perform numerical simulations to evaluate the performance of the proposed algorithms in MIMO secrecy SWIPT systems through numerical simulations. For simplicity, it was assumed that $N_{\mathrm{h}, k}=N_{\mathrm{h}}, \eta_{k}=\eta, \xi_{k}=$ $1, \forall k, p=q=\rho$, and $\sigma_{\mathrm{d}}^{2}=\sigma_{\mathrm{e}}^{2}=1$. In particular, we will examine the case in which the QoS requirements are

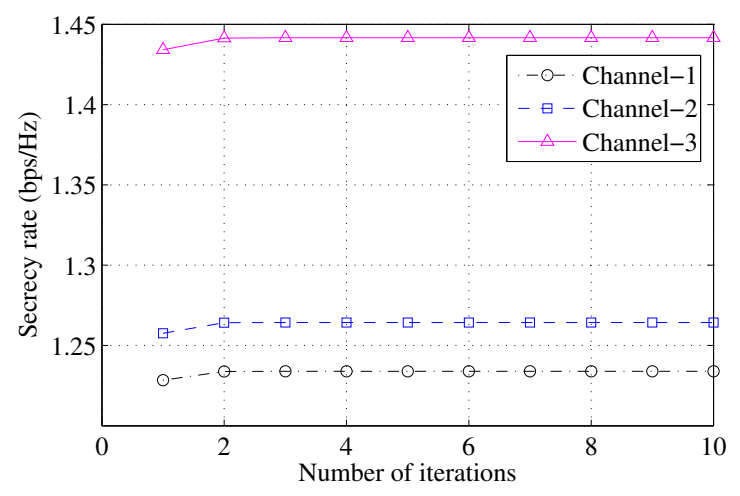

Fig. 2. Convergence of the outage-constrained secrecy rate maximization problem with $N_{\mathrm{T}}=8, N_{\mathrm{d}}=N_{\mathrm{e}}=N_{\mathrm{h}}=K=2$, and $\eta=-5(\mathrm{~dB})$.

such that each user is provided with an outage probability of at most $10 \%$; i.e., $\rho=0.1$, unless otherwise specified. All the estimated channel matrices are generated as zero-mean circularly symmetric independent and identically distributed complex Gaussian random variables and the TGn path-loss model for urban cellular environment is adopted considering a path-loss exponent of 2.7 [22]. In addition, we define the error covariance matrix of the eavesdropper's channel as $\mathbf{R}_{\mathrm{e}}=$ $\varepsilon_{\mathrm{e}}^{2} \mathbf{I}_{N_{\mathrm{T}} N_{\mathrm{e}}}$ and that of the $k$ th ER as $\mathbf{R}_{\mathrm{h}, k}=\varepsilon_{\mathrm{h}, k}^{2} \mathbf{I}_{N_{\mathrm{T}} N_{\mathrm{h}, k}}$ where $\varepsilon_{\mathrm{e}}^{2}, \varepsilon_{\mathrm{h}, k}^{2}$ represent the corresponding channel error variances. We also assume that $\varepsilon_{\mathrm{e}}^{2}=\varepsilon_{\mathrm{h}, k}^{2}=\varepsilon^{2}, \forall k$. All simulation results are averaged over 500 independent channel realizations, unless explicitly mentioned.

We start the performance analysis of the proposed convex restriction based alternating algorithm by evaluating its convergence speed. Fig. 2 shows the convergence of the secrecy rate maximization problem in different channel realization with an initial $\tilde{\mathbf{Q}}_{\mathrm{I}}=\mathbf{I}_{N_{\mathrm{e}}}$ for $N_{\mathrm{T}}=8, N_{\mathrm{d}}=N_{\mathrm{e}}=N_{\mathrm{h}}=K=2$, and $\eta=-5(\mathrm{~dB})$. It can be observed that the proposed algorithm achieves a fast convergence in various channel scenarios.

In the next example, we examine the secrecy rate performance of the proposed safe convex restriction algorithm against transmit power. For comparison purpose, we introduce the very basic sub-optimal scheme named plain maximum ratio transmission (Plain MRT) which ignores the presence of Eve. Hence, the transmit covariance matrix is defined as $\mathbf{Q}_{\mathrm{I}}=\left(\frac{P_{\mathrm{T}}}{\left\|\mathbf{H}_{\mathrm{d}}\right\|_{\mathrm{F}}^{2}}\right) * \mathbf{H}_{\mathrm{d}} \mathbf{H}_{\mathrm{d}}^{H}$ along the direction of the IR's channel. Fig. 3 plots the secrecy rates of the methods against the average transmit power constraint $P_{\mathrm{T}}$ for $N_{\mathrm{T}}=8$ and 10 . Other parameters were set as $N_{\mathrm{d}}=N_{\mathrm{e}}=N_{\mathrm{h}}=K=2$, and $\eta=-5(\mathrm{~dB})$. Interestingly, the proposed scheme yields almost 3 bits per channel use secrecy rate gain over the baseline scheme at $5 \mathrm{~dB}$ transmit power. Since the secrecy rate $R_{\mathrm{S}}$ in (6) is an increasing function the transmit power $\operatorname{tr}\left(\mathbf{Q}_{\mathrm{I}}\right)$, one can notice identical reflections in the results of Fig. 3 with increasing $P_{\mathrm{T}}$. Also, it is no surprise that a larger number of transmit antennas $\left(N_{\mathrm{T}}=10\right)$ yields a higher secrecy rate since the additional antennas can provide additional spatial DoF.

Finally, we analyze the energy harvesting performance of 


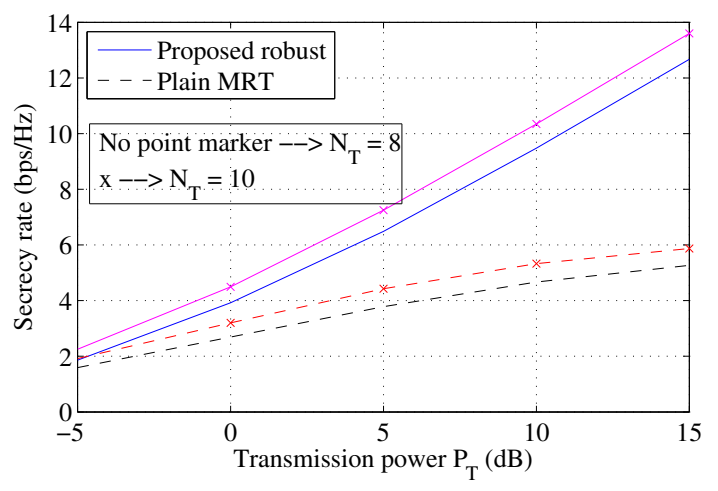

Fig. 3. Secrecy rate versus transmit power $P_{\mathrm{T}}$ with $N_{\mathrm{d}}=N_{\mathrm{e}}=N_{\mathrm{h}}=$ $K=2$, and $\eta=0(\mathrm{~dB})$.

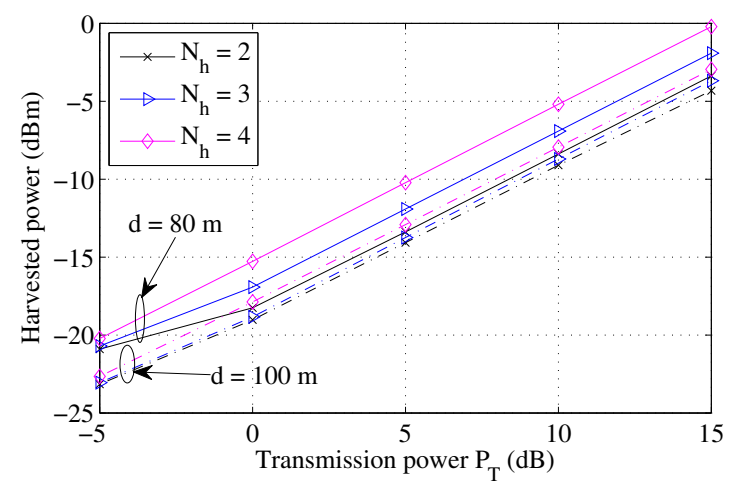

Fig. 4. Transmit power versus EH outage requirement $\eta$ with $N_{\mathrm{T}}=8$, $K=3, L=N_{\mathrm{e}}=2$, and $R=3,5(\mathrm{bps} / \mathrm{Hz})$

the ERs based on the proposed robust design with $N_{\mathrm{T}}=8$, $N_{\mathrm{d}}=N_{\mathrm{e}}=N_{\mathrm{h}}=K=2$, and $\eta=0(\mathrm{~dB})$. The results in Fig. 4 indicate that the harvested power increases in line with transmit power. However, an increased distance (100 meter) results in adverse effect on the harvested energy due to the propagation path-loss.

\section{CONCLUSIONS}

In this paper, we investigated probabilistically constrained secrecy rate maximization problem for SWIPT in a MIMO wiretap system where all the nodes are equipped with multiple antennas. Since the problem is non-convex with intractable probabilistic constraints, we proposed a convex safe approximation based robust transmit precoding algorithm with imperfect CSI. In particular, we transformed the probabilistic constraints involving log and det functions into SOC constraints which are easier to handle. Then we showed that a robust safe solution can be obtained through solving two convex sub-problems in an alternating fashion. Numerical simulations were carried out to demonstrate the effectiveness of the proposed approach over conventional baseline schemes.

\section{REFERENCES}

[1] X. Lu, P. Wang, D. Niyato, D. I. Kim, and Z. Han, "Wireless networks with RF energy harvesting: A contemporary survey," IEEE Commun. Surveys \& Tutorials, vol. 17, pp. 757-789, 2015.

[2] R. Zhang and C. K. Ho, "MIMO broadcasting for simultaneous wireless information and power transfer," IEEE Trans. Wireless Commun. vol. 12, pp. 1989-200, May 2013.

[3] M. R. A. Khandaker and K.-K. Wong, "SWIPT in MISO multicasting systems," IEEE Wireless Commun. Letters, vol. 3, pp. 277-280, June 2014.

[4] - "QoS-based multicast beamforming for SWIPT," in Proc. IEEE SECON Workshop Energy Harvesting Commun., Singapore, June 30July 03, 2014, pp. 62-67.

[5] J. Xu, L. Liu, and R. Zhang, "Multiuser MISO beamforming for simultaneous wireless information and power transfer," in Proc. IEEE Int. Conf. Acoust., Speech, Signal Process. (ICASSP), 2013, pp. 47544758 .

[6] L. Liu, R. Zhang, and K.-C. Chua, "Secrecy wireless information and power transfer with MISO beamforming," IEEE Trans. Signal Process., vol. 62, pp. 1850-1863, Apr. 2014.

[7] M. R. A. Khandaker and K.-K. Wong, "Masked beamforming in the presence of energy-harvesting eavesdroppers," IEEE Trans. Inf. Forensics and Security, vol. 10, pp. 40-54, Jan. 2015.

[8] H. Xing, L. Liu, and R. Zhang, "Secrecy wireless information and power transfer in fading wiretap channel," IEEE Trans. Vehicular Technology, vol. 65, pp. 180-190, Jan. 2016.

[9] M. R. A. Khandaker and K.-K. Wong, "Robust secrecy beamforming with energy-harvesting eavesdroppers," IEEE Wireless Commun. Letters, vol. 4, pp. 10-13, Feb. 2015.

[10] R. Feng, Q. Li, Q. Zhang, and J. Qin, "Robust secure transmission in MISO simultaneous wireless information and power transfer system," IEEE Trans. Veh. Technol., vol. 64, pp. 400-405, Jan. 2015.

[11] S. Wang and B. Wang, "Robust secure transmit design in MIMO channels with simultaneous wireless information and power transfer," IEEE Signal Process. Letters, vol. 22, pp. 2147-2151, Nov. 2015.

[12] Z. Chu, Z. Zhu, M. Johnston, and S. L. Goff, "Simultaneous wireless information power transfer for MISO secrecy channel," IEEE Trans. Veh. Technol., to appear, 2016.

[13] A. Khisti and G. W. Wornell, "Secure transmission with multiple antennas II: The MIMOME wiretap channel," IEEE Trans. Inf. Theory, vol. 56, pp. 5515-5532, Nov. 2010.

[14] S. Christensen, R. Agarwal, E. Carvalho, and J. Cioffi, "Weighted sumrate maximization using weighted MMSE for MIMO-BC beamforming design," IEEE Trans. Wireless Commun., vol. 7, pp. 4792-4799, Dec. 2008.

[15] K.-Y. Wang, A. M.-C. So, T.-H. Chang, W.-K. Ma, and C.-Y. Chi, "Outage constrained robust transmit optimization for multiuser MISO downlinks: Tractable approximations by conic optimization," IEEE Trans. Signal Process., vol. 62, pp. 5690-5705, Nov. 2014.

[16] Q. Li, W.-K. Ma, and A. M.-C. So, "A safe approximation approach to secrecy outage design for MIMO wiretap channels," IEEE Signal Process. Letters, vol. 21, pp. 118-121, Jan. 2014.

[17] Z. Chu, K. Cumanan, Z. Ding, M. Johnston, and S. L. Goff, "Robust outage secrecy rate optimizations for a MIMO secrecy channel," IEEE Wireless Commun. Letters, vol. 4, Feb. 2015.

[18] I. Bechar, "A Bernstein-type inequality for stochastic processes of quadratic forms of Gaussian variables," available online at http: / / arxiv.org/abs/0909.3595, Sep. 2009.

[19] S. Boyd and L. Vandenberghe, Convex Optimization. Cambridge, U. K.: Cambridge University Press, 2004.

[20] S. Janson, "Large deviations for sums of partly dependent random variables," Random Struct. Algorithms, vol. 24, pp. 234-248, May 2004.

[21] S.-S. Cheung, A. M.-C. So, and K. Wang, "Linear matrix inequalities with stochastically dependent perturbations and applications to chanceconstrained semidefinite optimization," SIAM J. Optim., vol. 22, pp. 1394-1430, 2012.

[22] IEEE P802.11 Wireless LANs, "TGn channel models," IEEE 802.1103/940r4, Tech. Rep., May 2004. 